\title{
Non-linear water waves generated by impulsive motion of submerged obstacles
}

\author{
N. I. Makarenko ${ }^{1,2}$ and V. K. Kostikov ${ }^{1,2}$ \\ ${ }^{1}$ Lavrentyev Institute of Hydrodynamics, 630090, Novosibirsk, Russia \\ ${ }^{2}$ Novosibirsk State University, 630090, Novosibirsk, Russia \\ Correspondence to: N. I. Makarenko (makarenko@hydro.nsc.ru) \\ Received: 14 August 2013 - Published in Nat. Hazards Earth Syst. Sci. Discuss.: 23 December 2013 \\ Revised: 7 March 2014 - Accepted: 11 March 2014 - Published: 8 April 2014
}

\begin{abstract}
A fully non-linear problem on unsteady water waves generated by an impulsively moving obstacle is studied analytically. Our method involves reduction of the Euler equations to the integral-differential system for the wave elevation together with normal and tangential fluid velocities at a free surface. Exact model equations are derived in explicit form in a case where an isolated obstacle is presented by a totally submerged elliptic cylinder. A small-time asymptotic solution is constructed for a cylinder which starts with constant acceleration from rest. It is demonstrated that the leading-order solution terms describe several wave regimes such as the formation of non-stationary splash jets by vertical rising or vertical submersion of the obstacle; the generation of diverging waves is also observed.
\end{abstract}

\section{Introduction}

In this paper, we consider the non-stationary problem on generation of intense water waves caused by localized underwater disturbances. There are several physical mechanisms of tsunami-type wave formation such as piston displacement of the ocean floor due to a submarine earthquake, lowfrequency hydro-elastic seismic oscillations, landslides and avalanches (Levin and Nosov, 2009). Long wave theory is very consistent in the case where the horizontal scale of a deformed bottom area is much bigger than the ocean depth. In this context, mathematical models describing the generation, propagation and run-up of tsunami waves were considered in many papers (see monographs by Pelinovsky, 1982, 1996 and papers by Pelinovsky and Mazova, 1992; Liu et al., 2003; Tinti and Tonini, 2005; and Dutykh and Dias, 2009). Non- linear energy transfer from elastic oscillations of a fluid layer to long gravitational waves were studied by Novikova and Ostrovsky (1982), Nosov and Skachko (2001), and Nosov et al. (2008).

Theoretical works on water waves generated by compact submerged sources (i.e. obstacles having relatively short horizontal scales) deal mostly with objects of a simple geometric form, such as a circular cylinder in 2-D motion and a sphere in the 3-D case. In addition, we neglect vorticity and viscosity to simplify the evaluation of pure inertial effects for the fluid flows generated by obstacles. Such a viewpoint is a common issue in marine hydrodynamics related to the problem on the interaction of submerged and floating bodies with the water surface (McCormick, 2010). This is due to the difficulty of mathematical formulations involving exact nonlinear boundary conditions at unknown free surfaces, as well as exact boundary conditions on the body surface or topographic conditions on the bottom surface. Linear problems on the obstacle modelled by singularity points in a viscous fluid were considered analytically by Lu and Chwang (2005).

There are a few analytical works related to the problem of non-linear interaction of submerged bodies with free surfaces of ideal fluids. A small-time non-stationary analytic solution was obtained by Tyvand and Miloh (1995a, b) and Makarenko (2003) in the presence of a circular cylinder, and by Pyatkina (2003) for a submerged sphere. This solution is in good agreement with experimental results by Greenhow and Moyo (1997). Our analysis uses reduction of Euler equations to the boundary integral-differential system which contains unknown variables on the free surface only. This method was developed by Ovsyannikov et al. (1985) for a non-linear water wave problem without submerged 
obstacles. Extension of this method exploits the idea suggested firstly by Wehausen (see Wehausen and Laitone, 1960) for stationary linear problems with submerged circular cylinders. It turns out that the Wehausen method can be generalized by using the Milne-Thomson transformation (see Milne-Thomson, 1996), even though the cylinder has a noncircular cross section (Makarenko, 2004). We apply this approach to study a fully non-linear water wave problem in the presence of a vertically moving plate modelled by a thin elliptic cylinder.

\section{Basic equations}

A potential 2-D flow of infinitely deep ideal fluid is considered on the coordinate system $O x y$ with a vertical $y$ axis, so a free surface $\Gamma(t)$ has a form $y=\eta(x, t)$ with the equilibrium level $y=0$. A compact plate-type solid obstacle is modelled here by a thin elliptic cylinder having a non-dimensional horizontal semi-axis $a$ and a vertical semi-axis $b(a \gg b)$ (see Fig. 1). This finite-size obstacle is supposed here to be totally submerged under a free surface. We suppose also that the cylinder moves without rotation, and the centre of its crosssection has a known trajectory $\mathbf{x}_{c}(t)=\left(x_{c}(t), y_{c}(t)\right)$.

Dimensionless formulation of the problem uses the initial depth of submergence $h_{0}$ of the centre as a length scale, the characteristic speed of cylinder $u_{0}$ as the velocity unit, the quantity $\rho u_{0}^{2}$ as the pressure unit (here $\rho$ is the fluid density), and the ratio $h_{0} / u_{0}$ as the time unit. Basic non-stationary Euler equations for the fluid velocity $\boldsymbol{u}=(U, V)$ and the pressure $p$ are

$U_{t}+U U_{x}+V U_{y}+p_{x}=0$,

$V_{t}+U V_{x}+V V_{y}+p_{y}=-\lambda$,

$U_{x}+V_{y}=0, \quad U_{y}-V_{x}=0$.

Here the constant $\lambda=g h_{0} / u_{0}^{2}$ is a square of the inverse Froude number defined by the gravity acceleration $g$. The boundary conditions at the unknown free surface $\Gamma(t)$ are

$\eta_{t}+U \eta_{x}=V, \quad p=0 \quad(y=\eta(x, t))$

and the condition on the surface of the obstacle $S(t)$ has the form

$\left(\boldsymbol{u}-\boldsymbol{u}_{\mathrm{c}}\right) \cdot \boldsymbol{n}=0$.

Here $\boldsymbol{n}$ is a unit normal to the boundary of the elliptic cylinder, and $\boldsymbol{u}_{\mathrm{c}}=\boldsymbol{x}_{\mathrm{c}}^{\prime}(t)$ is the speed of the submerged body at the present time $t$. In general, such an obstacle could be simply modelled by moving a finite-length horizontal plate of vanishing thickness. However, we consider here the smooth obstacle in order to avoid singularity of solution at the edge points of the curve $S(t)$. We suppose that the fluid is at rest at infinity

$U, V \rightarrow 0, \quad \eta \rightarrow 0 \quad(|\mathbf{x}| \rightarrow \infty)$.

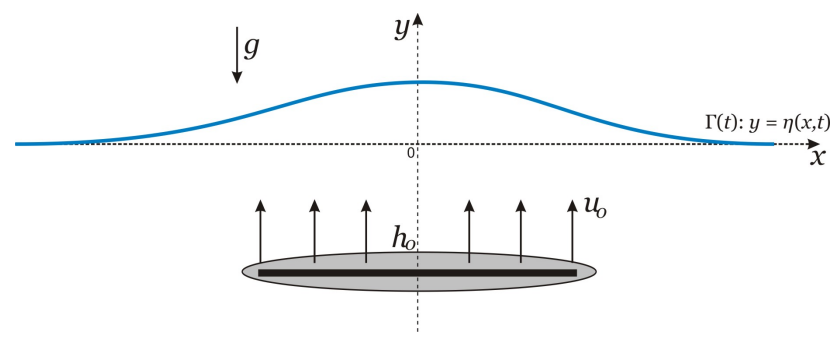

Fig. 1. Scheme of the motion.

We suppose also that the initial velocity field satisfies the compatibility conditions

$U_{0 x}+V_{0 y}=0, \quad U_{0 y}-V_{0 x}=0$,

$\left(\boldsymbol{u}_{0}-\boldsymbol{u}_{\mathrm{c}}(0)\right) \cdot \boldsymbol{n}_{0}=0$.

These matching conditions are fulfilled obviously for initial data $U_{0}=V_{0}=0$ and $x_{\mathrm{c}}^{\prime}(0)=y_{\mathrm{c}}^{\prime}(0)=0$ when the obstacle starts moving from rest.

\section{Boundary integral-differential equations}

The main difficulty in solving the problem formulated above arises from the unknown free surface with non-linear boundary condition. Therefore we reduce Eqs. (1)-(3) to the system of integral-differential equations being one-dimensional with respect to spatial variables. Let

$u(x, t)=\left.\left(U+\eta_{x} V\right)\right|_{y=\eta(x, t)}, \quad v(x, t)=\left.\left(V-\eta_{x} U\right)\right|_{y=\eta(x, t)}$

be tangential and normal fluid velocities at the free surface $\Gamma(t)$. Excluding the pressure from momentum Eqs. (1) and (2) we obtain under both conditions (Eq. 4) the non-linear evolution system for $\eta, u, v$ which has the form

$\eta_{t}=v, \quad u_{t}+\frac{1}{2} \frac{\partial}{\partial x} \frac{u^{2}-2 \eta_{x} u v-v^{2}}{1+\eta_{x}^{2}}+\lambda \eta_{x}=0$.

The differential Eq. (8) should be complemented by an integral equation at the free boundary $\Gamma(t)$ appearing due to the Cauchy-Riemann Eq. (3) for the fluid velocity components $U$ and $V$. The derivation procedure uses the analyticity of a complex velocity $F=U-i V$ with respect to the complex variable $z=x+i y$. We refer here to Silverman (1973) for a related fact of complex analysis. Basically, the function $F(z, t)$ satisfies the Cauchy integral formula

$2 \pi i F(z, t)=\int_{\Gamma(t)} \frac{F(\zeta, t) \mathrm{d} \zeta}{\zeta-z}+\int_{S(t)} \frac{F(\zeta, t) \mathrm{d} \zeta}{\zeta-z}$,

where $z$ is an arbitrary point belonging to the flow domain. Let $z_{\mathrm{c}}(t)=x_{\mathrm{c}}(t)+i y_{\mathrm{c}}(t)$ be the symmetry centre of the submerged body bounded by the elliptic contour $S(t)$ in a complex $z$ plane. We introduce an auxiliary $\tau$ plane in order to 
use a conformal map by evaluation of the integral on curve $S(t)$ in Eq. (9). Note that the Joukowski function

$\zeta=z_{\mathrm{c}}(t)+\tau+\frac{a^{2}-b^{2}}{4 \tau}$

maps conformally an exterior domain $|\tau| \geqslant r$ of the circle having the radius $r=(a+b) / 2$ onto an exterior domain of the ellipse $S(t)$. The inverse map is given by the analytic branch of the function

$\tau(\zeta ; t, a, b)=\frac{1}{2}\left(\zeta-z_{\mathrm{c}}(t)+\sqrt{\left(\zeta-z_{\mathrm{c}}(t)\right)^{2}-a^{2}+b^{2}}\right)$

which conforms to an exterior domain of the ellipses with given semi-axes $a$ and $b$ onto the exterior domain $|\tau| \geqslant r$ of the circle of the radius $r$. This function together with the Cauchy kernel satisfies the following useful relation:

$$
\frac{1}{\zeta-z}=\frac{\tau^{\prime}(z)}{\tau(\zeta)-\tau(z)}+\frac{\tau_{1}^{\prime}(z)}{\tau(\zeta)-\tau_{1}(z)}
$$

where $\tau_{1}(z)=\left(a^{2}-b^{2}\right) /(4 \tau(z))$. In addition, if the complex variable $\zeta$ belongs to the elliptic contour $S(t)$, we also have the relation

$$
\frac{1}{\tau(\zeta)-\tau(z)}=-\frac{1}{\tau(z)} \overline{\left(\frac{\tau(\zeta)}{\tau(\zeta)-\tau_{*}(z)}\right)}
$$

where $\tau_{*}=r^{2} / \bar{\tau}$ is the inversion image of $\tau$ with respect to the circle $|\tau|=r$, and the bar denotes the complex conjugate.

Using these relations enables us to exclude the integration around the obstacle surface $S(t)$ by transforming to integrals on a free surface $\Gamma(t)$ due to the residue theorem. The derivation is straightforward, but we refer to the paper by Makarenko (2004) which involves calculations of related integrals. By this way, Eq. (9) takes the form

$$
\begin{aligned}
2 \pi i F(z, t) & =\int_{\Gamma(t)} \frac{F(\zeta, t) \mathrm{d} \zeta}{\zeta-z}+r^{2}\left(\overline{\int_{\Gamma} \frac{F(\zeta, t) \mathrm{d} \zeta}{\tau(\zeta)-\tau_{*}(z)}}\right. \\
& \left.-k \int_{\Gamma(t)} \frac{F(\zeta, t) \mathrm{d} \zeta}{\tau(\zeta)-k \overline{\tau_{*}(z)}}\right) \frac{\tau^{\prime}(z)}{\tau^{2}(z)} \\
& +2 \pi i r^{2}\left(z_{\mathrm{c}}^{\prime}(t)-k \overline{z_{\mathrm{c}}^{\prime}(t)}\right) \frac{\tau^{\prime}(z)}{\tau^{2}(z)}
\end{aligned}
$$

where the parameter $k$ is given by the formula

$k=\frac{a-b}{a+b}$.

The limit value $k=0$ corresponds to the circular obstacle of the radius $r=a=b$. In this case, the conform map $\tau(\zeta ; t, a, b)$ reduces to the linear function $\tau=\zeta-z_{\mathrm{c}}(t)$, so Eq. (12) couples the Cauchy integral on $\Gamma(t)$ together with their Milne-Thomson transformation. This combination of integrals on $\Gamma(t)$ provides automatically the validity of the boundary condition (Eq. 5) on the curve $S(t)$ due to the Milne-Thomson circle theorem. In that sense, Eq. (12) with $a \neq b$ gives the extension of the Milne-Thomson theorem for the elliptic contour $S(t)$. Note that a residual term with $z_{\mathrm{c}}^{\prime}(t)$ appearing on the right-hand side of Eq. (12) is similar to the fluid velocity term for the elliptic cylinder surrounded by infinite flow.

The real-valued form of an integral equation arises if we set $z=x+i \eta(x, t)$ in Eq. (12), taking into account a discontinuity jump of the Cauchy integral at the free surface $\Gamma(t)$. By that way, combination of the real and imaginary parts of Eq. (12) yields a singular integral equation for the functions $\eta, u$, and $v$ having the form

$$
\pi v(x)+\int_{-\infty}^{\infty} A(x, s) v(s) \mathrm{d} s=\int_{-\infty}^{+\infty} B(x, s) u(s) \mathrm{d} s+f(x),
$$

where the real-valued kernels $A$ and $B$ can be split by the formulas

$$
A=A_{\Gamma}+r^{2} A_{S}, \quad B=B_{\Gamma}+r^{2} B_{S} .
$$

Here the kernels

$$
A_{\Gamma}(x, s)+i B_{\Gamma}(x, s)=\frac{i\left[1+i \eta_{x}(x)\right]}{x-s+i[\eta(x)-\eta(s)]}
$$

correspond to a perfect non-linear water wave problem without any submerged obstacle, and the additional kernels

$$
\begin{gathered}
A_{S}(x, s)+i B_{S}(x, s)=\left\{\frac{1}{\overline{\tau(s+i \eta(s))}-\overline{\tau_{*}(x+i \eta(x))}}\right. \\
\left.+\frac{k}{\tau(s+i \eta(s))-k \overline{\tau_{*}(x+i \eta(x))}}\right\}\left\{\frac{i}{\tau(x+i \eta(x))}\right\}_{x}
\end{gathered}
$$

appear due to the presence of the obstacle. The extra term $f$ in Eq. (13) has the form

$$
f(x)=r^{2} \operatorname{Re}\left\{\frac{2 \pi i\left(k \overline{z_{\mathrm{c}}^{\prime}(t)}-z_{\mathrm{c}}^{\prime}(t)\right)}{\tau(x+i \eta(x))}\right\}_{x} .
$$

The time variable $t$ is omitted everywhere in Eq. (13) because it appears in this integral equation only as a parameter. More precisely, this independent variable is presented here in explicit form only by known functions $z_{\mathrm{c}}(t)$ and $z_{\mathrm{c}}^{\prime}(t)$. So, the time $t$ is coupled implicitly with the unknown functions $v(x, t), u(x, t)$, and $\eta(x, t)$. They should satisfy the boundary integral-differential system Eq. (8), Eq. (13) which is equivalent to the fully non-linear water wave problem in the presence of a submerged elliptic obstacle. This system determines immediately the exact wave elevation and fluid velocity at the free surface. 


\section{Small-time solution}

We consider here in more detail the initial stage of the fluid motion beginning from rest $\eta(x, 0)=u(x, 0)=0$. It is supposed that the obstacle starts smoothly with zero speed $z_{\mathrm{c}}^{\prime}(0)=0$ at the time moment $t=0$. Therefore we also have $v(x, 0)=0$ immediately from Eq. (13). Thus, we can seek a solution as a power series

$$
\begin{aligned}
& \eta(x, t)=t \eta_{1}(x)+t^{2} \eta_{2}(x)+t^{3} \eta_{3}(x)+\ldots, \\
& u(x, t)=t u_{1}(x)+t^{2} u_{2}(x)+t^{3} u_{3}(x)+\ldots, \\
& v(x, t)=t v_{1}(x)+t^{2} v_{2}(x)+t^{3} v_{3}(x)+\ldots .
\end{aligned}
$$

Coefficients $\eta_{n}$ and $u_{n}$ are evaluated via $v_{n}$ by recursive formulas following from evolution Eq. (8). Namely, we have $\eta_{1}=u_{1}=u_{2}=0$ and

$\eta_{n}=\frac{1}{n} v_{n-1} \quad(n=2,3, \ldots)$,

$u_{3}=\frac{1}{6}\left(v_{1}^{2}-\lambda v_{1}\right)_{x}, \quad u_{4}=\frac{1}{4}\left(v_{1} v_{2}\right)_{x}-\frac{1}{12} \lambda v_{2 x}, \quad \ldots$

Consequently, integral Eq. (13) for normal velocity $v$ reduces to the set of recursive integral equations for coefficients $v_{n}$ $(n=1,2, \ldots)$ having the form

$$
\pi v_{n}(x)+r^{2} \int_{-\infty}^{+\infty} A_{S}^{(0)}(x, s) v_{n}(s) \mathrm{d} s=\varphi_{n}(x) .
$$

Here the kernel $A_{S}^{(0)}$ is a known function collecting lowerorder terms of the kernels $A$ and $B$ from Eq. (13) with substituted power series of $\eta$,

$A_{S}^{(0)}(x, s)=\operatorname{Re}\left\{\left(\frac{1}{\overline{\tau(s)}-\overline{\tau_{*}(x)}}+\frac{k}{\tau(s)-k \overline{\tau_{*}(x)}}\right)\left(\frac{i}{\tau(x)}\right)_{x}\right\}$.

The same is true for the right-hand side function $\varphi_{1}$ which represents the lowest-order term of the function $f$ from Eq. (13). In contrast, the functions $\varphi_{n}(n \geqslant 2)$ depend nonlocally and non-linearly on the coefficients $v_{1}, \ldots, v_{n-1}$.

Further simplification is possible by the perturbation procedure involving appropriate small parameters. It was rigorously proved in the paper by Makarenko (2003) that for a circular cylinder having a small radius $r=a=b$ the solution of Eq. (8), Eq. (13) is of the order $r^{2}$. Subsequently, the terms to be neglected are of the order $r^{4}$ by this approximation. This fact explains especially why the leading-order solution is in very good agreement with fully non-linear numerical solutions and laboratory experiments; even the submergence depth is comparable with the radius of the cylinder (up to the bound $r \sim 0.5$ ). Keeping this in mind, we apply a similar perturbation method in the case of a thin elliptic obstacle with a sufficiently small horizontal semi-axis $a$ and vanishing vertical semi-axis $b=0$. The simplest explicit formula for a symmetric wave solution appears in the case of vertical rising or vertical submersion of an obstacle with constant acceleration $w$. By that, we can originally take $u_{0}=\sqrt{w h_{0} / 2}$ as the velocity unit, so the trajectory of the obstacle takes the dimensionless form as $x_{\mathrm{c}}(t)=0, y_{\mathrm{c}}(t)=-1 \pm t^{2}$. As a result, we obtain a small-time asymptotic solution of accuracy $\sim a^{4}$ which has a free surface elevation $\eta$ as follows:

$\eta(x, t)=Q_{x}(x ; a) t^{2}+P_{x x}(x ; a)\left( \pm \frac{\lambda}{6}-1\right) \frac{t^{4}}{2}+O\left(t^{6}\right)$.

Here the function $P(x ; a)$ is even in $x$, the function $Q(x ; a)$ is odd, and both these elementary functions are defined as $x \geqslant 0$ by the formulas

$$
\begin{aligned}
& P(x ; a)=-2+\sqrt{2} \sqrt{\sqrt{\left(x^{2}-a^{2}-1\right)^{2}+4 x^{2}}-x^{2}+a^{2}+1}, \\
& Q(x ; a)=2 x-\sqrt{2} \sqrt{\sqrt{\left(x^{2}-a^{2}-1\right)^{2}+4 x^{2}}+x^{2}-a^{2}-1} .
\end{aligned}
$$

Note that only the term with $\lambda$ in Eq. (15) has the sign depending on the direction of motion. Namely, sign "plus" in this formula corresponds to the lift of the obstacle, and "minus" should be taken for submersion. In any case, the terms combined in the solution to Eq. (15) allow the water surface $y=\eta(x, t)$ to oscillate near the equilibrium level $y=0$.

Such a simple analytic solution allows an easy comparison of the wave regimes (at least qualitatively) by a variation of basic parameters. Figures 2-5 demonstrate typical wave profiles generated due to the vertical motion of accelerated obstacle. For all the pictures, the centre of the elliptic body is placed initially at the point $x=0, y=-1$ as $t=0$. Dashed and solid lines are used to show the water surface corresponding to the position of obstacle at the instant.

Early stages of the wave motion by slow and fast rising of the submerged plate are compared in Fig. 2 and Fig. 3. Rapidity of the motion is regulated here by a unique dimensionless parameter $\lambda$. In both cases, the obstacles are shown at the same submergence depths reached by the same dimensionless time moments $t=0.6$ and $t=0.8$ (but not at the same physical time!). It is clear that the wave generator has enough time to produce the system of diverging waves before the moderately accelerated obstacle comes near the free surface (see Fig. 2). In contrast, fast motion of the obstacle leads to the formation of an added fluid layer which results in strong displacement of the free surface concentrated over the plate (Fig. 3). Fig. 4 shows that the volume of this inertial fluid layer depends strongly on the horizontal size of an obstacle. This effect can be explained by the fact that the inertial fluid layer forms over the rigid obstacle during the time period of the order $t^{2}$ (Makarenko, 2003). Finally, Fig. 5 demonstrates an example of free surface flow generated by vertical submersion of the obstacle. This picture reveals significant distinction of this case from the flow regimes mentioned above, namely, one can see here that the splash jet forms only after initial depression of a free surface caused by sinking of the obstacle. 


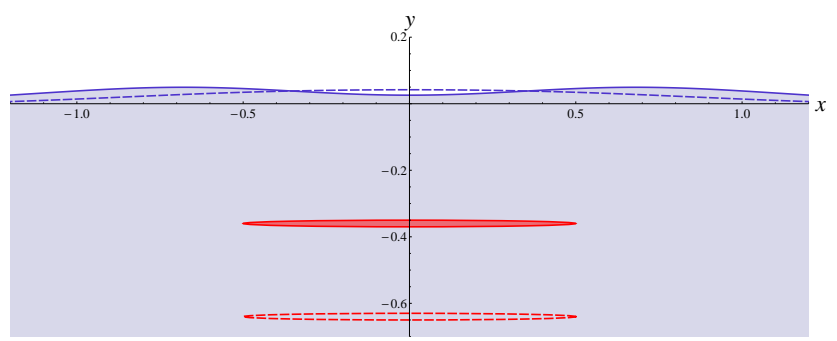

Fig. 2. Formation of diverging waves by slow rising of the obstacle: $a=0.5, \lambda=15$.

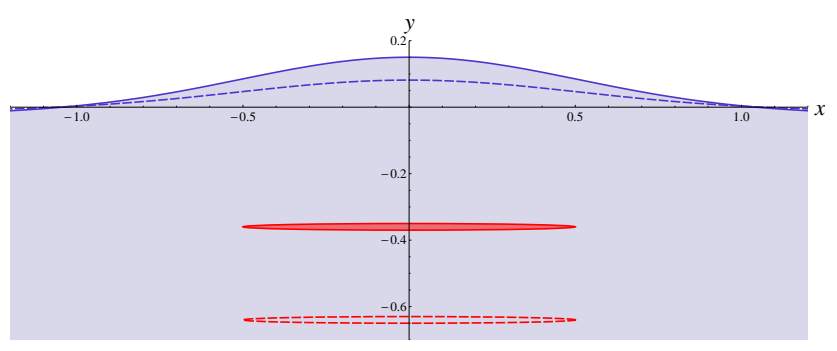

Fig. 3. Formation of inertial fluid layer by fast rising of the obstacle: $a=0.5, \lambda=15$.

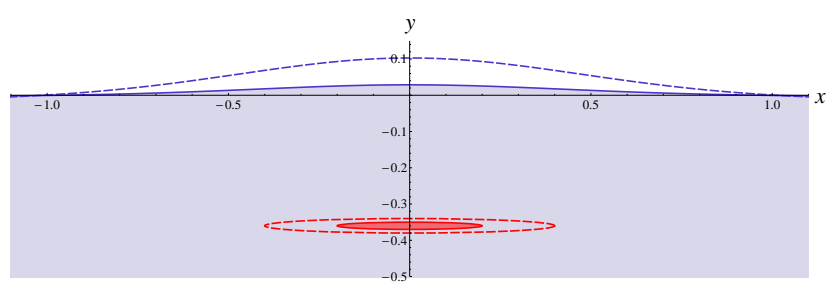

Fig. 4. Formation of added fluid mass by the obstacles with different semi-axes: $a=0.2$ (solid lines) and $a=0.4$ (dashed lines); $\lambda=5$, $t=0.8$.

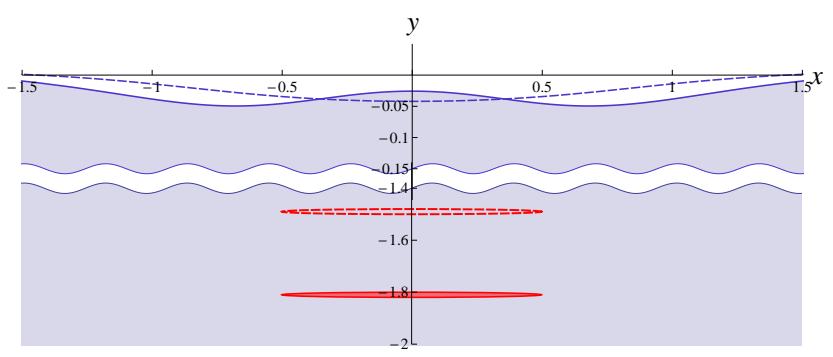

Fig. 5. Formation of splash jets by submersion of the obstacle: $a=$ $0.5, \lambda=3$. Position at time $t=0.6$ (dashed lines) and $t=0.8$ (solid lines).

\section{Conclusions}

In this paper, the non-linear water wave problem is considered in the presence of a thin horizontal elliptic cylinder submerged beneath a free surface of deep fluid. An equivalent system of integral-differential equations for the wave elevation and the velocity components at the free surface is formulated. In this framework, we study theoretically the piston mechanism of the generation of hazardous tsunami-type waves due to a fast deformation of a compact bottom area. A small-time asymptotic solution is constructed by elementary functions for a vertically accelerated obstacle started from rest. Magnitude of acceleration is involved in the mathematical model as the control parameter, so the initial stage of the flow is simulated with high asymptotic accuracy. Variation of basic parameters demonstrates that the constructed approximate solution realistically reproduces the main features of non-stationary wave processes.

Acknowledgements. This work was supported by the Russian Foundation for Basic Research (grant no. 12-01-00671), program RAS (project no. 23.2) and interdisciplinary project SB RAS no. 117.

Edited by: A. Toffoli

Reviewed by: two anonymous referees

\section{References}

Dutykh, D. and Dias, F.: Energy of tsunami waves generated by bottom motion, P. Roy. Soc. A., 465, 725-744, 2009.

Greenhow, M. and Moyo, S.: Water entry and exit of horizontal circular cylinder, Philos. T. Roy. Soc. Lond. A., 355, 551-563, 1997.

Levin, B. and Nosov, M.: Physics of tsunamis, Springer, Dordrecht, 2009.

Liu, P. L.-F., Lynett, P., and Synolakis, C. E.: Analytical solutions for forced long waves on a sloping beach, J. Fluid Mech., 478, 101-109, 2003.

Lu, D. Q. and Chwang, A. T.: Unsteady free surface waves due to a submerged body moving in a viscous fluid, Phys. Rev., E71, 066303, 2005.

Makarenko, N. I.: Nonlinear interaction of submerged cylinder with free surface, JOMAE Trans. ASME, 125, 72-75, 2003.

Makarenko, N. I.: Nonlinear water waves in the presence of submerged elliptic cylinder, Proc. 23rd Int. Conf. on Offshore Mech. and Arctic Engineering OMAE'04, 20-25 June, 2004, Vancouver, Canada, Paper OMAE-51413, 2004.

McCormick, M. E.: Ocean engineering mechanics with applications, Cambridge, Cambridge Univ. Press, 2010.

Milne-Thomson, L. M.: Theoretical hydrodynamics, Dover Publ., New York, 1996.

Nosov, M. A. and Skachko, S. N.: Nonlinear tsunami generation mechanism, Nat. Hazards Earth Syst. Sci., 1, 251-253, doi:10.5194/nhess-1-251-2001, 2001. 
Nosov, M. A., Kolesov, S. V., and Denisova, A. V.: Contribution of nonlinearity in tsunami generated by submarine earthquake, Adv. Geosci., 14, 141-146, doi:10.5194/adgeo-14-141-2008, 2008.

Novikova, L. E. and Ostrovsky, L. A.: On an acoustic mechanism of tsunami wave generation, Oceanology, 22, 693-697, 1982.

Ovsyannikov, L. V., Makarenko, N. I., Nalimov, V. I., Liapidevskii, V. Yu., Plotnikov, P.I., Sturova, I. V., Bukreev, V. I., and Vladimirov, V. A.: Nonlinear problems of the theory of surface and internal waves (Russian), Nauka, Novosibirsk, 1985.

Pelinovsky, E. N.: Nonlinear dynamics of tsunami waves (Russian), Inst. Appl. Physics Press, Gorky, 1982.

Pelinovsky, E. N.: Tsunami wave hydrodynamics (Russian), Inst. Appl. Physics Press, Nizhny Novgorod, 1996.

Pelinovsky, E. N. and Mazova, K. N.: Exact solutions of nonlinear problems of tsunami wave run-up onslopes with different profiles, Nat. Hazards, 6, 227-249, 1992.
Pyatkina, E. V.: Small-time expansion of wave motion generated by a submerged sphere, J. Appl. Mech. Techn. Phys., 44, 32-43, 2003.

Silverman, R. A.: Complex analysis with applications, Dover Publ., N.J. Prentice Hall, New York, 1973.

Tinti, S. and Tonini, R.: Analytical evolution of tsunamis induced by near-shore earthquakes on a constant-slope ocean, J. Fluid Mech., 535, 33-64, 2005.

Tyvand, P. A. and Miloh, T.: Free-surface flow due to impulsive motion of a small submerged circular cylinder, J. Fluid Mech., 286, 67-101, 1995a.

Tyvand, P. A. and Miloh, T.: Free-surface flow generated by a small submerged circular cylinder starting from the rest, J. Fluid Mech., 286, 103-116, 1995b.

Wehausen, J. V. and Laitone, E. V.: Surface Waves, Handbuch der Physik, 9, 446-778, Springer Verlag, Berlin, 1960, 